\title{
Investigation of the Effects of Low-Energy Plasma Treatment on Amorphous Carbon and Graphene Support Films for Cryo-Electron Microscopy
}

\author{
MJ Campin ${ }^{1}$, M Stumpf $^{1}$ and PE Fischione ${ }^{1}$ \\ 1. E.A. Fischione Instruments, Inc., Export, PA, USA. \\ * Corresponding author:mj_campin@fischione.com
}

The availability of direct-detection cameras and advanced image processing software has greatly improved the speed and ease of data collection and analysis in structural biology [1]. This has allowed single particle cryo-electron microscopy (cryo-EM) to develop into a key technique for the analysis of macromolecular structures. However, cryo-EM is still a rapidly growing field with unique challenges, including the techniques used for specimen preparation. Transmission electron microscopy (TEM) of biological specimens requires the use of support films deposited on standard specimen grids. For cryo-EM, mesh copper or gold grids with an amorphous carbon (am-C) supporting material is commonly used because it induces adsorption of particles to the grid and minimizes the effect of the support on TEM images [2, 3]. Unfortunately, the methods commonly used to manufacture am-C supports result in variability of the performance between grids [3]. For example, whether the grid is weakly or strongly hydrophilic or hydrophobic can result in the concentration of particles in the grid holes being lower or higher than was in the applied droplet [4]. Also, because the quality of the results relies on obtaining high quality specimens that produce consistent, accurate data, it is important to use high quality substrates that react to droplets in a consistent manner so that results accurately represent the original material; this minimizes valuable microscope and analysis time spent on specimens mounted on poor substrates. One approach to obtaining good substrates is to use a low-energy argon-oxygen plasma to modify the support film so that it becomes hydrophilic, which allows the droplet to spread uniformly over the film [4]. Another approach is to use an entirely different support such as graphene, which is an ideal substrate for imaging cryo-EM specimens, and use a low-energy hydrogen plasma to remove surface contamination and make the support hydrophilic [4].

Standard 400-mesh copper grids with ultra-thin amorphous carbon (am-C) support on lacey carbon were initially used to test the ability to create hydrophilic grids. For these experiments, the NanoClean plasma cleaner [Fischione Instruments] was used to process up to 10 grids simultaneously using a multi-grid specimen holder [Fischione Instruments], which allows for plasma treatment from either one side or both sides of the grid. A gas mixture of $5 \% \mathrm{O}_{2} / 95 \%$ Ar and a power setting of 10 watts were used to allow for maximum controllability of the process. Figure 1 shows optical images of a water droplet placed on a grid before plasma treatment. The droplet placed on the grid before plasma treatment has a large contact angle and did not spread across the entire grid, whereas the droplet placed on the grid after plasma treatment has a much smaller contact angle and spread across the entire grid, which indicates an increase in hydrophilicity. Figure 2 shows TEM images of the amorphous carbon support acquired on a Tecnai TF30 TEM [Thermo Fisher Scientific] at $300 \mathrm{kV}$. Figure $2 \mathrm{a}$ shows the grid before plasma treatment, Figure $2 \mathrm{~b}$ shows the grid after 3 minutes of double-sided plasma treatment, and Figure $2 \mathrm{c}$ shows the grid after 6 minutes of singlesided plasma treatment. These TEM images show no significant degradation of the am-C support, even after extended plasma treatment times. Additional investigations of low-energy plasma treatment of am-C supports using argon and hydrogen-argon gas mixtures and plasma treatment of graphene supports using hydrogen-argon gas mixtures is in progress. 


\section{References:}

[1] G McMullan et al., Ultramicroscopy 147 (2014), p. 156.

[2] M Cheung et al., Journal of Structural Biology 182 (2013), p. 51.

[3] CJ Russo and LA Passmore, Nature Methods 11 (2014) p. 649.

[4] LA Passmore and CJ Russo, "Specimen Preparation for High-Resolution Cryo-EM" in Methods in Enzymology, 579, ed. RA Crowther (Academic Press, Burlington).
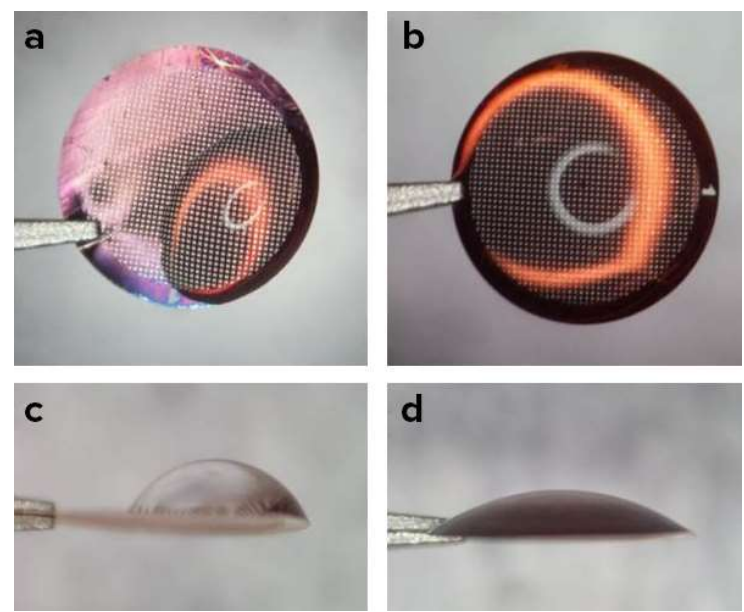

Figure 1. A water droplet placed on the grid before plasma cleaning (a, c) did not spread across the entire grid, which indicates that the grid is hydrophobic. In contrast, the water droplet placed on the grid after plasma cleaning $(b, d)$ spread across the entire grid and had a much smaller contact angle, which indicates that the grid is hydrophilic.
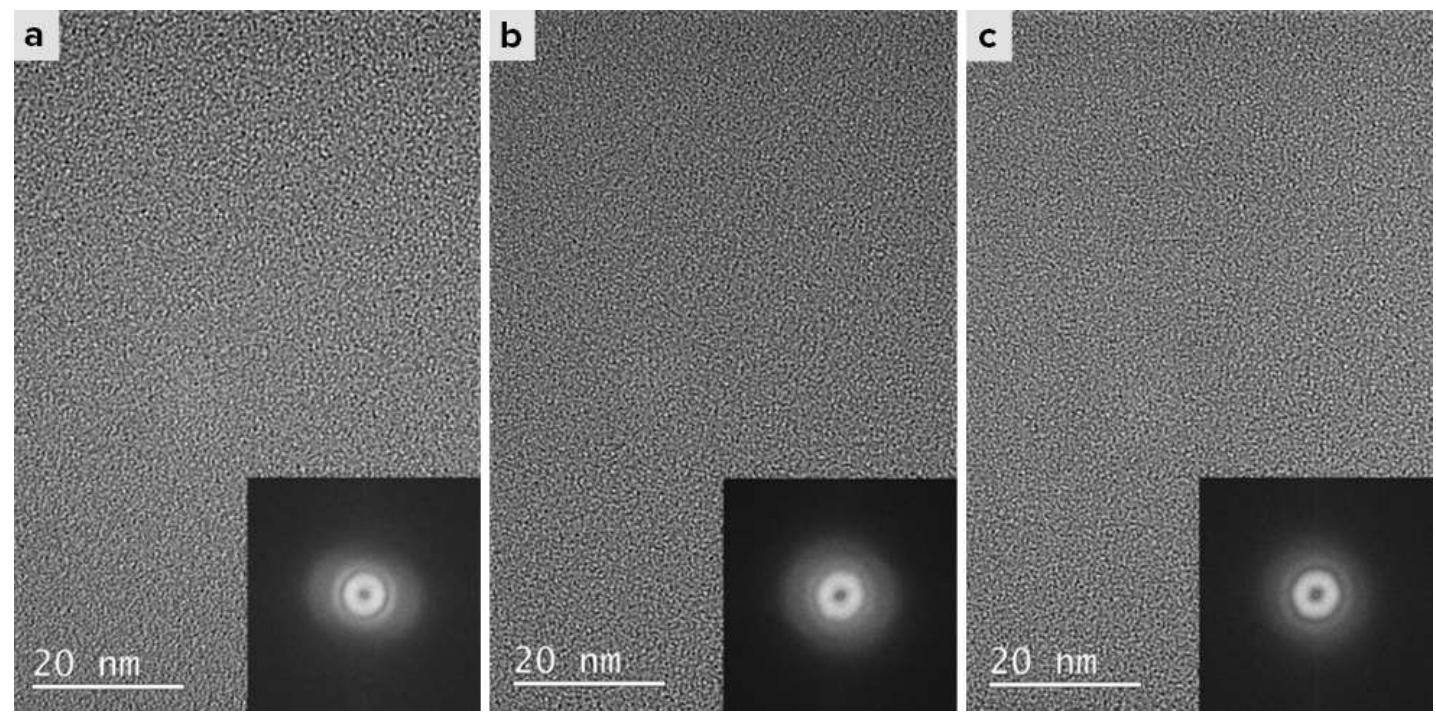

Figure 2. HRTEM images of the support film before plasma treatment (a), after double-sided plasma treatment (b) and after single-sided plasma treatment (c). Fast-Fourier transforms (FFTs) of the HRTEM images are inset. No significant change to the am-C film was noted after plasma treatment. 